\title{
Therapeutic effect of mesenchymal stem cells in rats with intracerebral hemorrhage: Reduced apoptosis and enhanced neuroprotection
}

\author{
SU-PING WANG, ZI-HAN WANG, DAO-YONG PENG, SHU-MIN LI, HONG WANG and XIAO-HONG WANG \\ The First Department of Neurology, Dalian Central Hospital, Dalian, Liaoning 116033, P.R. China
}

Received February 19, 2012; Accepted June 21, 2012

DOI: $10.3892 / \mathrm{mmr} .2012 .997$

\begin{abstract}
Stem cell transplantation has been used to improve neural function in intracerebral hemorrhage (ICH). However, reports on bone marrow-derived mesenchymal stem cell (MSC) transplantation in $\mathrm{ICH}$ are limited. We aimed to explore the therapeutic effect and related mechanisms by transplantation of MSCs in rats with $\mathrm{ICH}$. An experimental rat $\mathrm{ICH}$ model was established by intrastriatal administration of collagenase. The rats were randomly divided to receive either rat MSCs or PBS solution intravenously. In addition, behavioral tests using the modified neurological severity score (mNSS) were performed following ICH. Immunohistochemistry was performed to detect the Brdu-labeled MSCs and the protein expression of caspase 2, NF200 and GFAP in neural tissues. Western blotting and ELISA were performed to measure the protein expression of Akt and bcl-2 or the protein content of G-CSF and BDNF. The MSC-transplanted group demonstrated better neural function on the mNSS test following ICH compared with the control group $(\mathrm{P}<0.05)$. The MSC-transplanted group also showed reduced hemorrhage volume at 24 and $72 \mathrm{~h}$ following ICH. In the perihematomal regions of rat brain with $\mathrm{ICH}$, a substantial number of Brdu-labeled MSCs were observed, and a high protein expression of caspase 3, NF200 and GFAP was found in the MSC-transplanted group. The protein content of Akt, Bcl-2, G-CSF and BDNF were all elevated by MSC transplantation. Intravenously transplanted MSCs are capable of improving functional recovery and restoring neurological deficits in experimental ICH. The mechanisms are associated with enhanced survival and differentiation
\end{abstract}

Correspondence to: Dr Su-Ping Wang, The First Department of Neurology, Dalian Central Hospital, No. 826 Southwest Road, Shahekou District, Dalian, Liaoning 116033, P.R. China

E-mail: wangsupingzhuren@sina.com

Key words: intracerebral hemorrhage, mesenchymal stem cells, neurogenesis, trophic factors of neural cells, and increased expression of anti-apoptotic proteins and trophic factors.

\section{Introduction}

Intracerebral hemorrhage ( $\mathrm{ICH})$ is a common subtype of stroke and produces severe neurological deficits in survivors (1), with a poor prognosis that is much worse than that for ischemic strokes of a similar size. Currently, medical therapy for patients with ICH is generally limited to supportive care or surgical evacuation of the hematoma, with variable and controversial efficacy.

One important response of the brain to various injuries is an increased production of endogenous neural cells, which are differentiated from resident neural stem cells (NSCs) in discrete brain regions. NSCs are induced to proliferate in response to brain injury and migrate into areas of brain damage (2). NSCs retain the capacity for proliferation and lesion-directed migration in adult patients with cerebral ischemia (3). This provides the possibility of functional neuronal transplantation through endogenous neurogenesis.

$\mathrm{ICH}$ and ischemic strokes share similar responses to NSCs in the adult human brain. Increased neurogenesis has been reported following ICH in rats and humans $(4,5)$, which resulted from the activation of NSCs in the adult human brain following subarachnoid hemorrhage $(\mathrm{SAH})$, thus contributing to the promotion of spontaneous recovery (6). Significant improvements in behavioral tests have been reported in animals with ICH following NSC transplantation (7), indicating a potentially therapeutic effect in humans. The difficulty in obtaining NSCs limits their clinical applications; other sources of stem cells are required for replacement therapy in ICH. Among the various types of stem cells, one of the most promising stem cell sources are bone marrow-derived mesenchymal stem cells (MSCs), as they are capable of differentiating into neurons (8) and can be obtained from the patient's own bone marrow (9). Previously, it was reported that MSCs are able to differentiate into neural cells in the rat brain following $\mathrm{ICH}$, with significantly improved neural function (10). In this study, we investigated the therapeutic effect of intravenous transplantation of MSCs following $\mathrm{ICH}$ in rats. Our data suggest that transplantation of MSCs induces neural differentiation and proliferation of neural cells in the rat brain following ICH. 


\section{Materials and methods}

Animals. Male Sprague-Dawley (SD) rats (12 weeks old) were used for the experiment. The experiments and procedures were approved by the local animal care committee of Dalian Central Hospital.

Isolation and culture of bone marrow-derived MSCs. Rats were sacrificed and placed in $70 \%$ alcohol for disinfection. Both femurs were dissected and the attached muscle and soft tissue were stripped. A 21-gauge needle was applied to aspirate the bone marrow, followed by flushes through the bone using a $1 \mathrm{ml}$ syringe filled with $2 \mathrm{ml}$ of DMEM medium (Gibco, Carlsbad, CA, USA) containing 10\% fetal bovine serum (FBS). Approximately $10 \times 10^{6}$ bone marrow cells were diluted with an equal volume of Ficoll separation medium for centrifugation at $400 \mathrm{x} g$ for $30 \mathrm{~min}$. The white film-like layer of mononuclear cells was removed and seeded at a density of $1 \times 10^{5} / \mathrm{ml}$ in a $150 \mathrm{~cm}^{2}$ tissue culture flask coated with collagen I (Becton Dickinson Labware, Oxford, UK), and cultured at $37^{\circ} \mathrm{C}$ in a humidified incubator with $5 \% \mathrm{CO}_{2}$. Every 3-4 days, the cultured cells were digested by $0.25 \%$ trypsin with a passage ratio of 1:2. After the third passages reached $90 \%$ confluence, the cells were collected and preserved for further study.

Characterization and labeling of MSCs. MSCs were collected from the tissue culture flasks following the third passage and centrifuged at $200 \mathrm{x}$ g for $5 \mathrm{~min}$. A single cell suspension of $0.5-1 \times 10^{6}$ cells (50 $\mu$ l PBS) was incubated with primary antibodies against CD90, CD29, CD45 or CD11bc for $40 \mathrm{~min}$ and then FITC-labeled secondary antibody for $30 \mathrm{~min}$. Flow cytometry was applied to evaluate cell fluorescence (Becton Dickinson). Prior to injection, MSCs were labeled with 5-bromo 2'deoxy-uridine (BrdU, $5 \mu \mathrm{M}$ ) (Sigma Chemical, St. Louis, MO, USA) for $12 \mathrm{~h}$.

Rat ICH model generation. Adult male SD rats $(n=12)$ weighing 270-320 g were used in the study. The study used collagenase to induce ICH (11). Briefly, after preoperative fasting for $12 \mathrm{~h}$ and water deprivation for $4 \mathrm{~h}$, intraperitoneal anesthesia was performed with $10 \%$ chloral hydrate. Animals were anesthetized with their head fixed on the stereotaxic instrument. A midline scalp incision was made, and the injection sites of the exposed skull were $0.5 \mathrm{~mm}$ anterior and $3.5 \mathrm{~mm}$ lateral to bregma (caudate nucleus). Using a syringe pump (Harvard Instruments), $0.4 \mathrm{U}$ type VII bacterial collagenase (Sigma C0773) in $2 \mu 1$ normal saline, or saline alone (vehicle) was infused slowly $(0.5 \mu \mathrm{l} / \mathrm{min})$ into the centre of the striatum. The needle was left in place for $2 \mathrm{~min}$ and withdrawn slowly, with the hole sealed by bone wax. At $24 \mathrm{~h}$ post-surgery, animals were anesthetized with overdose chloral hydrate and sacrificed by decapitation. The brain was rapidly extracted and slices of cortex and basal ganglia were made. The brain tissue was fixed with $10 \%$ formaldehyde or homogenized for use in subsequent experiments.

Experimental groups. One hour following ICH, the rats were randomly divided into two groups, which were injected with $1 \mathrm{ml}$ PBS (ICH group) or $1 \mathrm{ml}$ PBS with cell suspension $\left(1 \times 10^{6}\right.$ MSCs) (ICH-MSCs group), respectively. Briefly, $1 \times 10^{6} \mathrm{MSCs}$ suspended in $1 \mathrm{ml}$ sterile PBS were injected into each animal in the ICH-MSCs group using a 27-G needle via the tail vein. Animals in the ICH group received an equal volume of PBS vehicle.

Behavioral testing of neurological deficits. To examine the effects of the transplanted MSCs on the neurological deficits of rats following $\mathrm{ICH}$, two investigators who were blinded to the treatment status performed the behavioral tests, with the modified neurological severity score (mNSS). The mNSS is a composite index of motor, sensory, beam balance and reflex tests (12). Injury in the right hemisphere striatum of ICH rats causes sensory and motor functional deficits, leading to elevated scores on motor, sensory and beam balance tests. The animals were monitored at 1, 3, 7, 14, 21 and 28 days following $\mathrm{ICH}$ (n=6/group).

Hemorrhage volume detection. Hemorrhage volume was quantified at 24 or $72 \mathrm{~h}$ via a spectrophotometric assay conducted as described previously (13). Measurements from perfused brains subjected to $\mathrm{ICH}$ were compared with the standard curve to obtain data in terms of hemorrhage volume ( $\mathrm{ml}$; $\mathrm{n}=6$ /group). Three duplicate experiments were performed in each group.

Tissue preparation and immunohistochemistry. On day 3, the anesthetized animals were sacrificed by decapitation $(n=6 /$ group). The brains were rapidly removed and placed in $4 \%$ paraformaldehyde at $4^{\circ} \mathrm{C}$ for $48 \mathrm{~h}$. Samples were incubated at $4^{\circ} \mathrm{C}$ overnight with primary antibodies against Brdu, caspase 3 (Santa Cruz Biotechnology, Santa Cruz, CA, USA), NF200, GFAP (Cell Signaling Technology Inc., Beverly, MA, USA) at concentrations of 1:100 (GFAP) and 1:200 (caspase 3, NF200). As the secondary antibody, immunoglobulin $\mathrm{G}$ conjugated with horseradish peroxidase (HRP) (Promega Biotechnology Co., Ltd., Beijing, China) was used for $30 \mathrm{~min}$ at $37^{\circ} \mathrm{C}$. Prior to dehydration and mounting, slides were counterstained with hematoxylin. The marker-specific cells were counted throughout the entire section (three sections per antibody staining) and then the total counts in these sections were converted into cell densities for quantitation.

Western blotting. Twenty-four hours following the induction of $\mathrm{ICH}$, the rats were sacrificed to extract the brain $(n=3 /$ group). Homogenates of the brain tissues in perihematomal areas were serially processed for western blotting. Cytosolic extracts $(50 \mu \mathrm{g})$ were resolved by $15 \%$ SDS-polyacrylamide gels (SDS-PAGE) and electrotransferred onto a nitrocellulose membrane. The extracts were then incubated with the primary antibodies anti-phospho-Akt (serine-473) (1:200) or anti-bcl-2 (1:200) for $1 \mathrm{~h}$, followed by secondary antibodies conjugated with HRP. Visualization was performed using the chemiluminescence method. $\beta$-actin was used as an internal reference.

Sandwich ELISA method for G-CSF and BDNF detection. Levels of granulocyte colony-stimulating factor (G-CSF) and brain-derived neurotrophic factor (BDNF) protein in the cytosolic extracts of brain tissue were assayed using the ChemiKine ELISA Kit (Chemicon, Temecula, CA, USA), according to the manufacturer's instructions. Briefly, the cytosolic extracts and standards were incubated for $2 \mathrm{~h}$, followed by washing with 
A

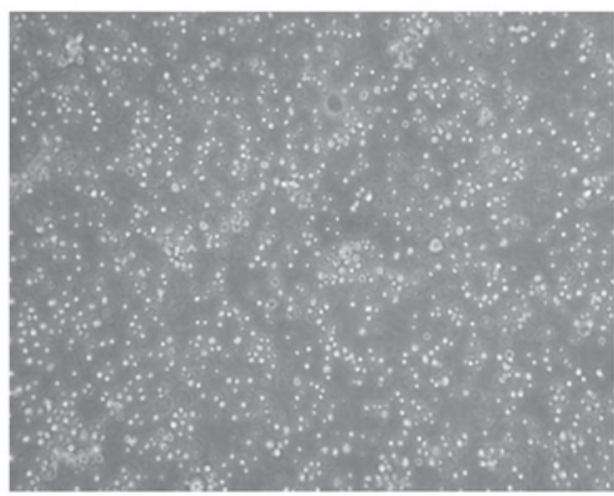

C

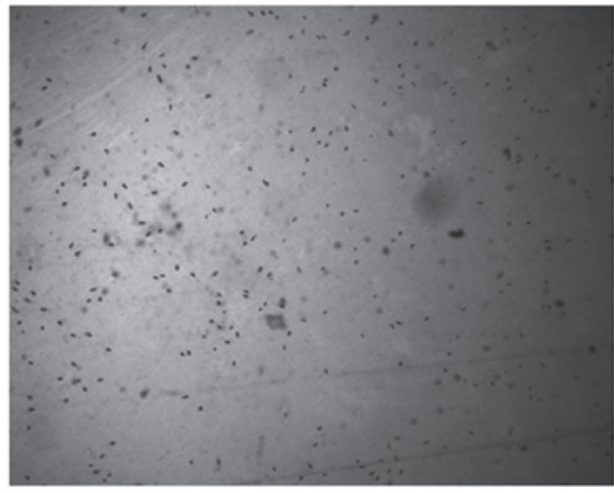

B

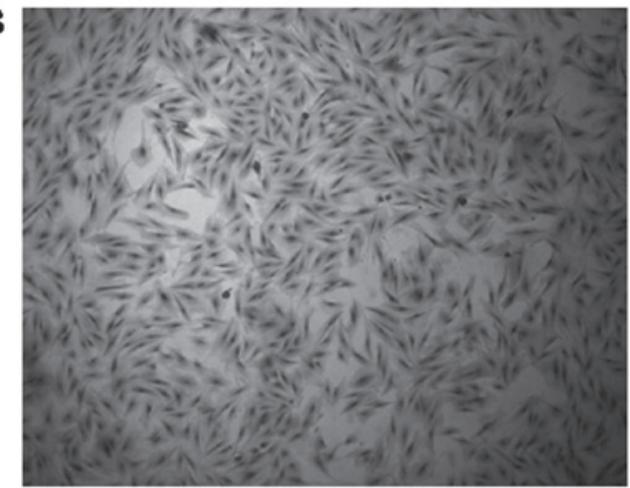

D
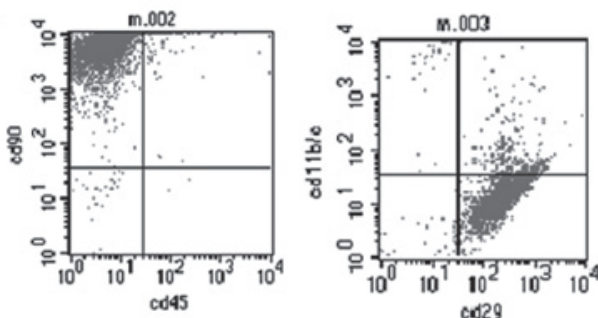

\begin{tabular}{|l|l|l|l|l|}
\hline & CD90 & CD45 & C D11b & CD29 \\
\hline MSCS $(\%)$ & $98.3 \pm 0.95$ & $0.91 \pm 0.24$ & $9.83 \pm 0.84$ & $98.1 \pm 0.26$ \\
\hline
\end{tabular}

Figure 1. Morphology and characterization of rat mesenchymal stem cells (MSCs). (A) A single cell suspension was isolated from the white film-like layer of mononuclear cells at the gradient interface. (B) Cultured cells reached $90 \%$ confluence and appeared homogeneous with the morphology of mesenchymal stem cells at passage 3 (H\&E staining). (C) Brdu was labeled to demonstrate the proliferating cells at passage 3. (D) Double labeling of CD90/CD45 and CD11b/ CD29 was performed to test the purity of MSCs from rat BM. BM, bone marrow.

buffer and incubation with anti-G-CSF or anti-BDNF polyclonal antibody at room temperature for $2 \mathrm{~h}$. After washing, the plates were incubated with anti-IgG antibody conjugated to HRP for $1 \mathrm{~h}$. The plates were then incubated in peroxidase substrate and tetramethylbenzidine solution to produce a color reaction, followed by the addition of $1 \mathrm{M} \mathrm{HCl}$ to stop the reaction. A microplate reader was applied to measure absorbance at $450 \mathrm{~nm}$. Values are expressed as percentages of the controls.

Statistical analysis. Data in this study were presented as the means \pm SD. Data were analyzed by Student's t-test if the data were normally distributed (Kolmogorov-Smirnov test; $\mathrm{P}>0.05$ ). When data were not normally distributed, the MannWhitney $\mathrm{U}$ test was used. $\mathrm{P}<0.05$ was considered to indicate a statistically significant difference.

\section{Results}

Characterization of bone marrow-derived MSCs and expression of surface markers. The bone marrow mononuclear cells were isolated using the Ficoll method to form a single cell suspension (Fig. 1A). The cells were seeded in tissue culture flasks. After $24 \mathrm{~h}$, some cells were adhered, extended and showed a short spindle appearance. The cells exhibited clone-like growth, with the majority of clones composed of the spindle, multi-shaped fibroblast-like cells. After 10-15 days, cells reached confluence in the bottom of the flasks. At passage 3, cells showed relatively uniform bipolar spindle-like morphology and bundles of fibers were arranged in parallel under the inverted microscope, with no hematopoietic cell hybrid clones (Fig. 1B). At passage 3,
Brdu was labeled to demonstrate the proliferation of MSCs and the cells with positive Brdu labeling indicated dividing cells (Fig. 1C).

MSCs were analyzed for cell surface antigens at passage 3. The results showed that MSCs were highly positive for CD90 $(98.1 \pm 0.26 \%)$ and CD29 $(98.3 \pm 0.95 \%)$. The positive rates for CD11bc $(9.83 \pm 0.84 \%)$ and CD45 $(0.91 \pm 0.24 \%)$ were low (Fig. 1D). Therefore, a high purity of MSCs could be reached.

MSC transplantation promotes functional recovery and reduces hemorrhage volume in rats with $\mathrm{ICH}$. $\mathrm{ICH}$ is usually characterized by behavioral deficits. The rats in the ICH-MSC group exhibited less profound neurological deficits and more neurological improvement on mNSS than the ICH-only group at 1 day and 3 days following the induction of $\mathrm{ICH}$, with an insignificant difference $(\mathrm{P}>0.05)$. The recovery of neurological functions was faster in the ICH-MSC group than in the ICH group, and the difference was statistically significant at 7, 14, 21 and 28 days ( $\mathrm{P}<0.05$; t-test) (Fig. 2A). Compared with the ICH group, transplantation of MSCs attenuated the hemorrhage volume by $52 \%$ at $24 \mathrm{~h}$ following $\mathrm{ICH}$, and by $61 \%$ at $72 \mathrm{~h}$ following $\mathrm{ICH}$ (Fig. 2B).

MSCs increase proliferation and reduce apoptosis of perihematomal cells. Brdu is a synthetic nucleoside and is commonly used in the detection of proliferating cells in living tissues. Brdu staining showed a high density of positively stained cells within the hemorrhage lesions (Fig. 3A). Quantitative analysis revealed differences in BrdU positively stained cells between the two 

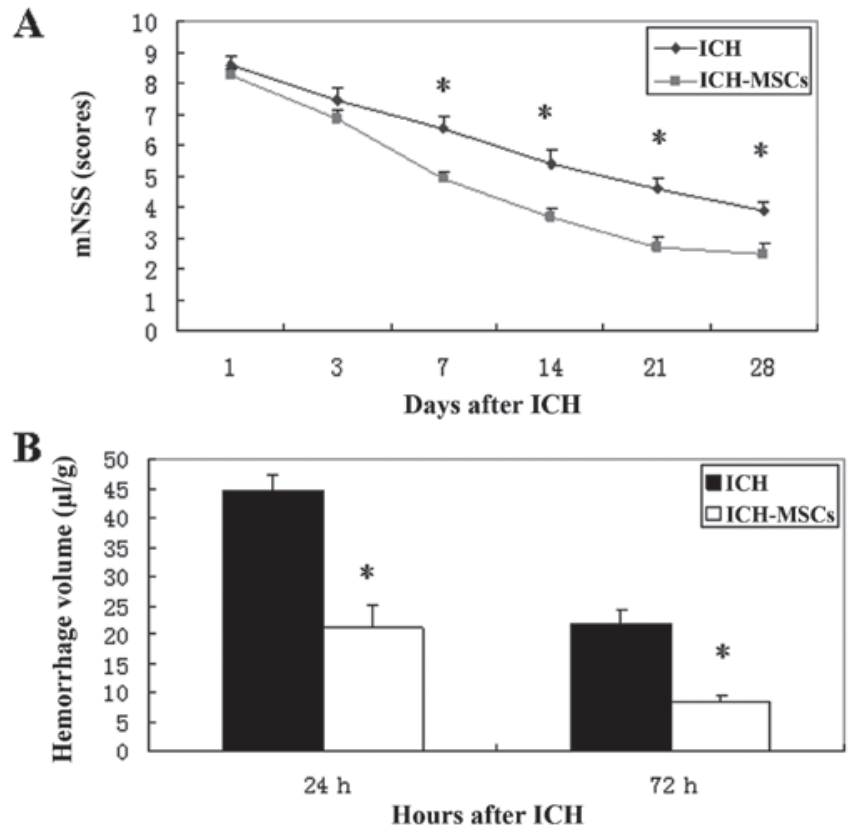

Figure 2. Behavioral test and hemorrhage volume. (A) The ICH-MSCs group exhibited fewer pronounced initial deficits, and it recovered better than the ICH group. (B) Transplanted MSCs reduced the hemorrhage volume by $52 \%$ at $24 \mathrm{~h}$ and by $61 \%$ at $72 \mathrm{~h}$ compared with the ICH group. "Significant differences between the ICH and ICH-MSC groups. ICH, intracerebral hemorrhage; MSCs, mesenchymal stem cells. groups. The ICH-MSCs group exhibited a significantly higher number of Brdu+ cells $\left(261.5 \pm 34.3\right.$ cells $\left./ \mathrm{mm}^{2}\right)$ than the ICH group $\left(1.9 \pm 0.75\right.$ cells $/ \mathrm{mm}^{2}$; $\mathrm{P}<0.01$; Fig. 3B). The ICH-MSCs group possessed significantly lower numbers of caspase $3+$ cells $\left(118.7 \pm 34.8\right.$ cells $\left./ \mathrm{mm}^{2}\right)$ than were observed in the ICH group $\left(264.9 \pm 31.5\right.$ cells $\left./ \mathrm{mm}^{2}\right)$ at 1 day following ICH $(\mathrm{P}<0.01$; Fig. $3 \mathrm{~B})$.

Neural protection and regeneration by MSCs. To determine the survival and differentiation of neural cells, immunohistochemistry was performed to detect the protein expression of NF200 and GFAP. The ICH-MSCs group demonstrated a higher expression of NF200 and GFAP than the ICH group (Fig. 4A). Quantitative analysis revealed differences between the two groups. The ICH-MSCs group exhibited a significantly higher number of NF200+ and GFAP+ cells (NF200+: $295.7 \pm 28.5$ cells $/ \mathrm{mm}^{2}$; GFAP+: $\left.178.8 \pm 32.6 \mathrm{cells} / \mathrm{mm}^{2}\right)$ than the ICH group (NF200+: $133.7 \pm 23.6$ cells $/ \mathrm{mm}^{2}$; GFAP+: $62.6 \pm 16.0$ cells $\left./ \mathrm{mm}^{2}\right)(\mathrm{P}<0.01$; Fig. $4 \mathrm{~B})$.

MSCs upregulated anti-apoptotic molecules, combined with the upregulation of G-CSF and BDNF. Western blotting demonstrated the upregulation of Akt and bcl-2 expression induced by MSC transplantation (Fig. 5A). Analysis of the protein band corresponding to Akt demonstrated a 3.6 0 0.6-fold increase in the cytosolic extracts of hemorrhagic brain tissue at $24 \mathrm{~h}$ after
$\mathbf{A}$

ICH

Caspase 3

B

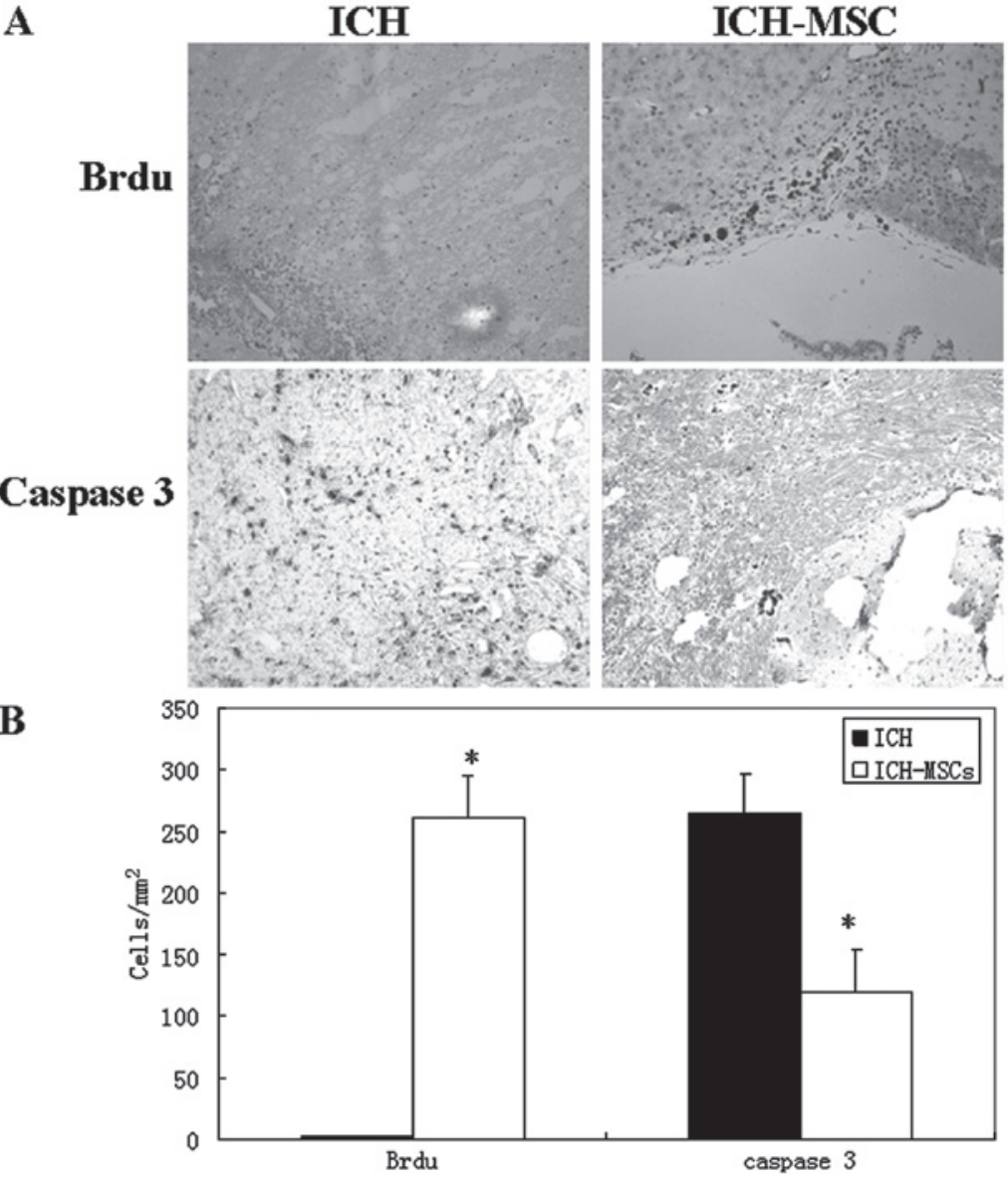

Figure 3. Histological analysis of proliferation and apoptosis. (A) Transplanted MSCs increased the number of Brdu+ cells and decreased the number of caspase 3+ cells in the perihematomal regions. (B) Quantification analysis demonstrated significant increases in the quantities of Brdu+ cells and reductions in the caspase $3+$ cells in the ICH-MSC group, compared with the ICH group. " $\mathrm{P}<0.05$ versus ICH (n=6; t-test). Representative images are shown. ICH, intracerebral hemorrhage; MSCs, mesenchymal stem cells. 
$\mathbf{A}$

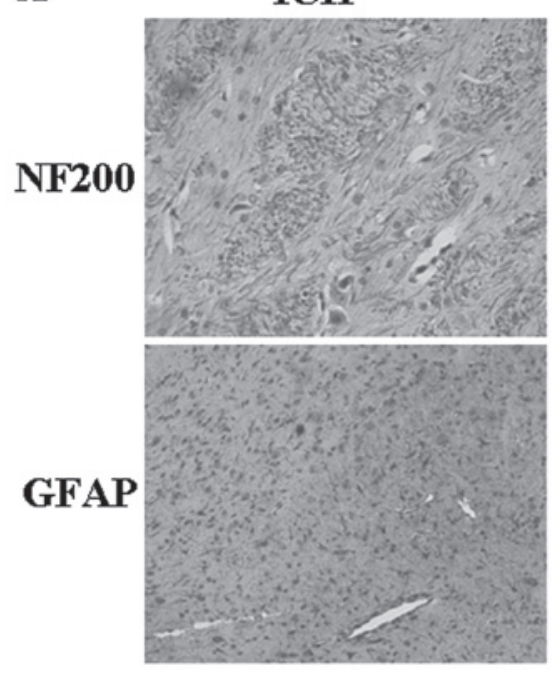

ICH-MSC
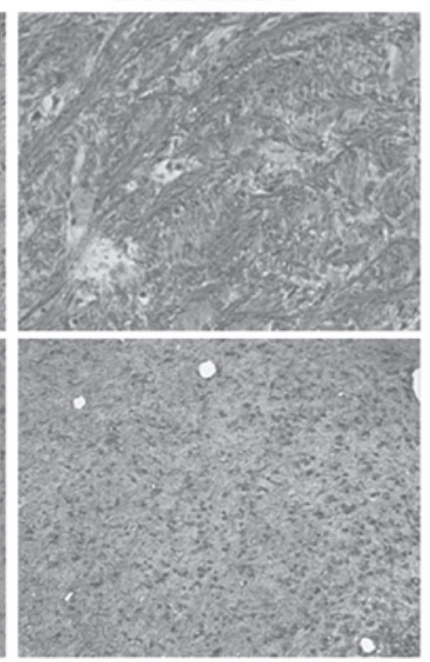

B

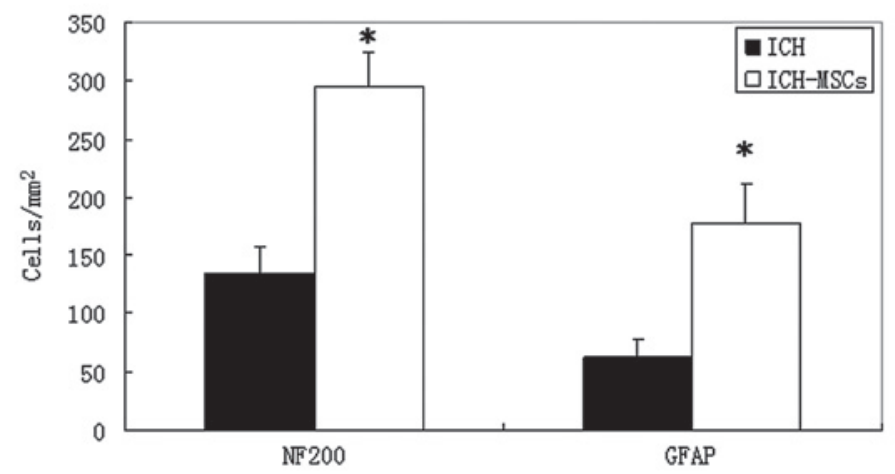

Figure 4. Neural protection and regeneration by MSCs. (A) Transplanted MSCs increased the number of NF200+ cells and GFAP+ in the perihematomal regions. (B) Quantification analysis demonstrated significant increases in the quantities of NF200+ and GFAP+ cells in the ICH-MSC group, compared with the ICH group. ${ }^{*} \mathrm{P}<0.05$ versus ICH ( $\mathrm{n}=6$; t-test). Representative images are shown. ICH, intracerebral hemorrhage; MSCs, mesenchymal stem cells.

the onset of ICH (Fig. 5B). The bcl-2 band showed a 5.9 \pm 0.8 -fold increase (Fig. 5C). The protein expression levels of Akt and bcl-2 were significantly increased in the ICH-MSC group compared to the ICH group ( $\mathrm{P}<0.01$, $\mathrm{t}$-test). The sandwich ELISA method was performed to detect the G-CSF and BDNF protein content in the cytosolic extracts of brain tissue. Significantly higher G-CSF and BDNF protein content was found in the ICH-MSC group than in the ICH group ( $\mathrm{P}<0.01$, t-test) (Fig. 5D).

\section{Discussion}

In this study, we undertook to characterize the therapeutic effects of MSCs and the mechanisms involved. Intravenous MSC transplantation during the hyperacute stage (at $1 \mathrm{~h}$ after $\mathrm{ICH})$ was able to reduce initial neurological deterioration, reduce hemorrhage volume and enhance functional recovery. MSCs provided neuroprotection, which was confirmed by the increased proliferation and reduced apoptosis of perihematomal cells. The transplanted MSCs also differentiated into neural cells, with an increased expression of anti-apoptotic proteins Akt and bcl-2 and trophic factors G-CSF and BDNF.

Our results suggest that earlier intravenous MSC administration is capable of reducing cerebral oedema following hemorrhagic stroke and improving neural function. ICH results in the mechanical disruption of brain tissue, including the neural and glial cells. Therefore, it was formerly thought that neural transplantation would be less likely to benefit this disorder. However, a previous report showed that intravenously transplanted NSCs are capable of entering the brain of mice with $\mathrm{ICH}$, and are able to survive, migrate and consequently improve functional recovery (14), indicating transplanted human NSCs are capable of restoring neurological deficits in experimental ICH. The scarce sources of NSCs limit their clinical application in ICH. MSCs were shown to possess stem cell features similar to NSCs, such as the self-renewal potential and multipotency, thus acting as potential candidate stem cells for replacement therapy in ICH. Our in vivo study shows that MSCs exhibit neuroprotective effects and promote functional recovery following CNS injury.

Early hematoma enlargement occurs in approximately $35 \%$ of patients with ICH within $3 \mathrm{~h}$ after onset, and the hemorrhage volume had been thought to be an independent predictor of poor outcome (15). Moreover, hematoma growth was also found to be a predictor of early neurological deterioration (16). Therefore, it has substantial clinical implications for early phase therapeutic interventions, particularly within $3 \mathrm{~h}$ of onset (17). In this study, we measured the hemorrhage volume at 24 and $72 \mathrm{~h}$, and found a significantly reduced hemorrhage volume at 24 and $72 \mathrm{~h}$ after ICH by MSCs. We also found that the hemorrhage volume was smaller at $72 \mathrm{~h}$ than at $24 \mathrm{~h}$, indicating its spontaneous absorption over time. Notably, transplanted MSCs are capable of accelerating this process. 
A

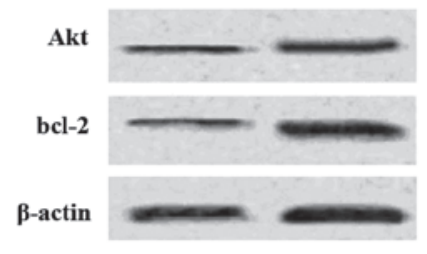

B

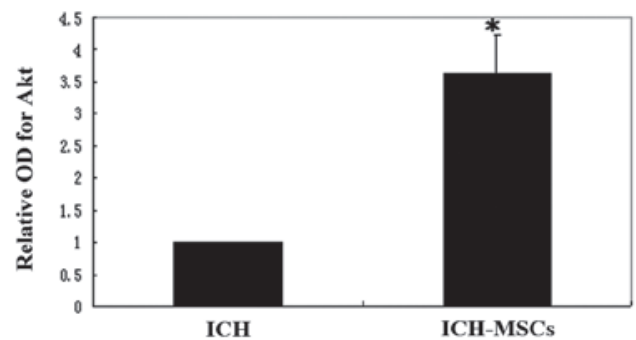

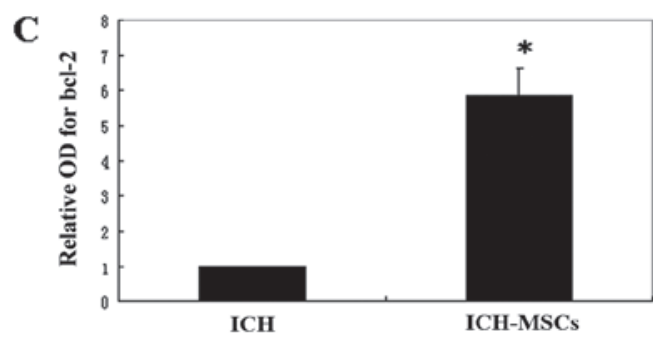

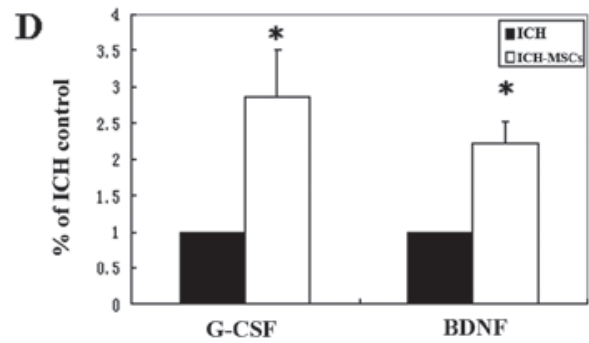

Figure 5. MSCs upregulated anti-apoptotic molecules, combined with the upregulation of G-CSF and BDNF. (A) Western blotting revealed the cytosolic fractions of Akt and bcl-2 expression. (B and C) Densitometric analysis of the band indicated an increase in the protein expression of Akt and bcl-2 in the ICH-MSC group compared with the ICH group $(\mathrm{P}<0.05)$. (D) Mean BDNF mRNA and protein levels in the frontal cortex from control and mood stabilizer-treated rats. The sandwich ELISA method was used to detect the G-CSF and BDNF protein levels. Data are expressed as a percentage of the control and are compared using a two-tailed, unpaired t-test (mean $\pm \mathrm{SD},{ }^{\mathrm{P}} \mathrm{P}<0.05$ ). The densitometric data are representative of three experiments. " $\mathrm{P}<0.01$ versus ICH ( $=3$; Mann-Whitney $\mathrm{U}$ test). Representative images are shown. ICH, intracerebral hemorrhage; G-CSF, granulocyte colony-stimulating factor; BDNF, brain-derived neurotrophic factor.

In $\mathrm{ICH}$, apoptosis is a prominent feature of neurotoxicity in the perihematomal region of brains with ICH (18). To investigate whether transplanted MSCs were able to reduce neurotoxicity and protect cells from apoptosis, immunohistochemistry was performed and a significantly reduced caspase 3 protein expression was found in perihematomal regions with MSC transplantation. Since transplanted MSCs attenuated the activities of caspase-3, which is an executive molecule in the common apoptotic pathway, we speculate that MSCs are capable of alleviating the intrinsic and extrinsic apoptotic pathways, which are activated in ICH. This suggests that the neuroprotective functions of MSCs may partly result from the elevated survival of neural cells in the perihematomal region of brains with ICH.

To explore whether the elevated survival of neural cells are caused by transplanted MSCs, we labeled MSCs with Brdu to track these cells in the brains of ICH rats. A large number of Brdu-labeled MSCs were found in perihematomal regions, which indicates that the transplanted MSCs are capable of entering the damaged brain tissue and initiating proliferation. Therefore, the stem cell properties of MSCs make them replicate or differentiate into neural cells in the rat brain following $\mathrm{ICH}$, which could explain the improved neural function after MSC transplantation (10).

The NSCs and neural progenitor cells (NPCs) reside in the subventricular zone (SVZ) in the adult mammalian brain. Neurogenesis occurs in response to brain injury induced by ICH $(4,5)$, and indicates possible spontaneous improvement following $\mathrm{ICH}$, which is partly confirmed by a declining curve over time in the mNSS test (Fig. 2A). In addition, transplanted MSCs promoted this process and improved neural function at late time points following ICH. To confirm the neurogenesis effect by transplanted MSCs, we detected the expression of phenotypic markers of mature neural cells in perihematomal regions. The results showed that the expression of NF200 and
GFAP markers were increased following MSC transplantation. The high molecular weight neurofilament marker NF200 is exclusively expressed on myelinated neurons (19), and GFAP was expressed on glial cells. Our result indicates enhanced neural differentiation after MSC transplantation in rats with ICH. The increased neural cells may come from endogenous neurogenesis or differentiation of MSCs. Previous reports showed that MSCs which were placed in the neurogenic area (hippocampus) generated neurons positive for NF200 (20), and GFAP+ glial cells were derived from transplanted MSCs in rats with experimental SAH (21). Those studies indicated that at least a substantial portion of increased neural cells originate from transplanted MSCs.

To explore the molecular mechanisms underlying improved survival and differentiation of neural cells, we measured the protein content of Akt, bcl-2, G-CSF and BDNF in the brain tissues of perihematomal regions, and found their expression was increased following MSC transplantation compared with the ICH group. Akt is a serine/threonine protein kinase and is crucial in cellular survival pathways by inhibiting apoptotic processes. Phosphorylation of Akt was reported to be enhanced after SAH (22), and this suggests that Akt may be involved in the survival pathway of injured brains after SAH. Moreover, overexpressed Akt1 in human NSCs provide neuroprotection and functional improvement in a mouse stroke model (23). In this study, we found that Akt was activated in rat brain with ICH by MSCs, indicating a similar survival pathway between NSCs and MSCs. Whether Akt overexpression is capable of promoting neuronal survival requires further investigation. The increased expression of the bcl-2 protein in this study was also confirmed by another report. It showed that the expression of bcl-2 was upregulated by Ginsenoside Rbeta1 treatment, which reduced neurological damage and apoptosis in the rat brain following SAH (24). This indicates that bcl-2 may be a common survival pathway in intracerebral hemorrhage induced 
by different therapeutics, and it therefore also plays a key role in the increased survival of neural cells by transplanted MSCs.

Our study found an increased G-CSF and BDNF protein content following MSC transplantation. Notably, the therapeutic effect of MSC transplantation may be mediated through increased G-CSF. It induces long-term sensorimotor recovery following $\mathrm{ICH}$ and is involved in reduced brain edema, inflammation, proliferation of NSCs and perihematomal cell death (25). We used bone marrow-derived MSCs in this study, which were reported to secrete more cytokines than umbilical cord blood-derived MSCs, indicating a higher possible therapeutic effect by MSCs from bone marrow than umbilical cord blood. BDNF is a type of growth factor that plays a key role in the growth and differentiation of central and peripheral nervous systems. BDNF is synthesized and expressed in neuronal and glial cells (26). Decreased BDNF concentrations are associated with intraventricular hemorrhage (27). Brain transplantation of BDNF-overexpressed human NSCs provided the differentiation and survival of NSCs and functional recovery of $\mathrm{ICH}$ animals (28). In addition to the BDNF expressed by neural cells, the increased BDNF in our study may also be secreted from grafted bone marrow-derived MSCs (29). Moreover, BDNF treatment also exerted an enforcing effect on MSCs and increased the BDNF production by MSCs, thus forming an autocrine regulation of MSCs (30).

In conclusion, we have provided convincing evidence to suggest that MSC transplantation exerts therapeutic effects in experimental $\mathrm{ICH}$ by improving neural function and reducing hemorrhage volume. The underlying mechanisms involve enhanced survival, the differentiation of neural cells and an elevated expression of anti-apoptotic proteins and trophic factors. Increased understanding of the mechanisms underlying neuroprotection by MSCs may lead to new treatments for $\mathrm{ICH}$.

\section{References}

1. Qureshi AI, Mendelow AD and Hanley DF: Intracerebral haemorrhage. Lancet 373: 1632-1644, 2009.

2. Nakatomi H, Kuriu T, Okabe S, Yamamoto S, Hatano O, Kawahara N, et al: Regeneration of hippocampal pyramidal neurons after ischemic brain injury by recruitment of endogenous neural progenitors. Cell 110: 429-441, 2002.

3. Jin K, Wang X, Xie L, Mao XO, Zhu W, Wang Y, et al: Evidence for stroke-induced neurogenesis in the human brain. Proc Natl Acad Sci USA 103: 13198-13202, 2006.

4. Masuda T, Isobe Y, Aihara N, Furuyama F, Misumi S, Kim TS, et al: Increase in neurogenesis and neuroblast migration after a small intracerebral hemorrhage in rats. Neurosci Lett 425 : 114-119, 2007.

5. Shen J, Xie L, Mao X, Zhou Y, Zhan R, Greenberg DA, et al: Neurogenesis after primary intracerebral hemorrhage in adult human brain. J Cereb Blood Flow Metab 28: 1460-1468, 2008.

6. Sgubin D, Aztiria E, Perin A, Longatti P and Leanza G: Activation of endogenous neural stem cells in the adult human brain following subarachnoid hemorrhage. J Neurosci Res 85: $1647-1655,2007$.

7. Li F, Liu Y, Zhu S, Wang X, Yang H, Liu C, et al: Therapeutic time window and effect of intracarotid neural stem cells transplantation for intracerebral hemorrhage. Neuroreport 18: 1019-1023, 2007.

8. Deng W, Obrocka M, Fischer I and Prockop DJ: In vitro differentiation of human marrow stromal cells into early progenitors of neural cells by conditions that increase intracellular cyclic AMP Biochem Biophys Res Commun 282: 148-152, 2001.

9. Dezawa M, Kanno H, Hoshino M, Cho H, Matsumoto N, Itokazu Y, et al: Specific induction of neuronal cells from bone marrow stromal cells and application for autologous transplantation. J Clin Invest 113: 1701-1710, 2004.
10. Zhang H, Huang Z, Xu Y and Zhang S: Differentiation and neurological benefit of the mesenchymal stem cells transplanted into the rat brain following intracerebral hemorrhage. Neurol Res 28: 104-112, 2006.

11. Rosenberg GA, Mun-Bryce S, Wesley M and Kornfeld M: Collagenase-induced intracerebral haemorrhage in rats. Stroke 21: 801-807, 1990.

12. Zhang L, Schallert T, Zhang ZG, Jiang Q, Arniego P, Li Q, et al: A test for detecting long-term sensorimotor dysfunction in the mouse after focal cerebral ischemia. J Neurosci Methods 117: 207-214, 2002

13. Lee ST, Chu K, Sinn DI, Jung KH, Kim EH, Kim SJ, et al: Erythropoietin reduces perihematomal inflammation and cell death with eNOS and STAT3 activations in experimental intracerebral hemorrhage. J Neurochem 96: 1728-1739, 2006.

14. Lee HJ, Kim KS, Kim EJ, Choi HB, Lee KH, Park IH, et al: Brain transplantation of immortalized human neural stem cells promotes functional recovery in mouse intracerebral hemorrhage stroke model. Stem Cells 25: 1204-1212, 2007.

15. Davis SM, Broderick J, Hennerici M, Brun NC, Diringer MN, Mayer SA, et al: Hematoma growth is a determinant of mortality and poor outcome after intracerebral hemorrhage. Neurology 66: 1175-1181, 2006.

16. Leira R, Davalos A, Silva Y, Gil-Peralta A, Tejada J, Garcia M, et al: Early neurologic deterioration in intracerebral hemorrhage: predictors and associated factors. Neurology 63: 461-467, 2004.

17. Mayer SA: Ultra-early hemostatic therapy for intracerebral hemorrhage. Stroke 34: 224-229, 2003.

18. Qureshi AI, Suri MF, Ostrow PT, Kim SH, Ali Z, Shatla AA, et al: Apoptosis as a form of cell death in intracerebral hemorrhage. Neurosurgery 52: 1041-1047, 2003.

19. Lawson SN and Waddell PJ: Soma neurofilament immunoreactivity is related to cell size and fibre conduction velocity in rat primary sensory neurons. J Physiol 435: 41-63, 1991.

20. Lepski G, Jannes CE, Strauss B, Marie SK and Nikkhah G: Survival and neuronal differentiation of mesenchymal stem cells transplanted into the rodent brain are dependent upon microenvironment. Tissue Eng Part A 16: 2769-2782, 2010.

21. Ali Khalili M, Anvari M, Hekmati-Moghadam SH, SadeghianNodoushan F, Fesahat F and Miresmaeili SM: Therapeutic benefit of intravenous transplantation of mesenchymal stem cells after experimental subarachnoid hemorrhage in rats. J Stroke Cerebrovasc Dis: Jan 29, 2011 [Epub ahead of print].

22. Endo H, Nito C, Kamada H, Yu F and Chan PH: Akt/GSK3beta survival signaling is involved in acute brain injury after subarachnoid hemorrhage in rats. Stroke 37: 2140-2146, 2006.

23. Lee HJ, Kim MK, Kim HJ and Kim SU: Human neural stem cells genetically modified to overexpress Akt1 provide neuroprotection and functional improvement in mouse stroke model. PLoS One 4: e5586, 2009

24. Li Y, Tang J, Khatibi NH, Zhu M, Chen D and Zheng W: Ginsenoside Rbetal reduces neurologic damage, is anti-apoptotic, and down-regulates p53 and BAX in subarachnoid hemorrhage. Curr Neurovasc Res 7: 85-94, 2010.

25. Zhang L, Shu XJ, Zhou HY, Liu W, Chen Y, Wang CL, et al: Protective effect of granulocyte colony-stimulating factor on intracerebral hemorrhage in rat. Neurochem Res 34: 1317-1323, 2009.

26. Radka SF, Holst PA, Fritsche M and Altar CA: Presence of brainderived neurotrophic factor in brain and human and rat but not mouse serum detected by a sensitive and specific immunoassay. Brain Res 709: 122-301, 1996.

27. Chouthai NS, Sampers J, Desai N and Smith GM: Changes in neurotrophin levels in umbilical cord blood from infants with different gestational ages and clinical conditions. Pediatr Res 53: 965-969, 2003.

28. Lee HJ, Lim IJ, Lee MC and Kim SU: Human neural stem cells genetically modified to overexpress brain-derived neurotrophic factor promote functional recovery and neuroprotection in a mouse stroke model. J Neurosci Res 88: 3282-3294, 2010.

29. Wilkins A, Kemp K, Ginty M, Hares K, Mallam E and Scolding N: Human bone marrow-derived mesenchymal stem cells secrete brain-derived neurotrophic factor which promotes neuronal survival in vitro. Stem Cell Res: Mar 27, 2009 [Epub ahead of print].

30. Choi YJ, Li WY, Moon GJ, Lee PH, Ahn YH, Lee G, et al: Enhancing trophic support of mesenchymal stem cells by ex vivo treatment with trophic factors. J Neurol Sci 298: 28-34, 2010. 